\title{
'Optimal nutrition'
}

\section{Optimal nutrition: an overview}

\author{
J. J. Strain \\ Northern Ireland Centre for Diet and Health, University of Ulster, Coleraine BT52 1SA, UK
}

The most problematic area that nutritionists have to address is how much is enough without becoming too much. The long-term consequences of too little or too much with respect to energy intakes for given energy expenditures can be outwardly readily recognized, but trying to define overnutrition or undernutrition with respect to other nutrients is fraught with difficulty. In theory, there should be many dietary regimens by which optimum nutrition and, therefore, optimum health and well-being can be achieved. Dietary recommendations, however, are based on nutrient intakes rather than food intakes, and there are many, probably intractable, challenges faced by those who are charged with producing dietary recommendations for populations. These challenges include the uncertainty caused by bioavailability issues such as nutrient-nutrient and nutrient-non-nutrient interactions, and other influences on nutrient requirements such as lifestyle factors, physiological state and genetic variability. It is probable that in the future diets will be prescribed on a personal basis, where nutritional needs are determined by individual genetic and metabolic characteristics.

In the meantime, emphasis is being increasingly placed on tailoring dietary recommendations to take account of the kinds and amounts of nutrients needed to optimize physiological and mental functions, and to minimize development of degenerative diseases. This changing emphasis takes cognizance of the potential multiple levels of nutrient activity (Combs, 1996). These levels could be defined as (a) the amount of nutrient needed to prevent overt deficiency disease, (b) the amount of nutrient needed to optimize biochemical, physiological and genetic functions associated with specific health benefits, (c) the pharmacological dose of nutrient needed to optimize non-traditional functions associated with specific health benefits and (d) the amount of nutrient that may lead to specific health hazards or toxic effects. It should be emphasized, however, that not all nutrients may have multiple levels of activity.

The recognition that some nutrients might be required in greater amounts than those simply needed to prevent overt deficiency disease indicates that the concept of nutritional essentiality should be revisited. The old concept of essentiality was based on observational and experimental findings that nutrients function to prevent deficiency disease. Perhaps a dietary factor should be considered as a 'nutrient' if it affects the metabolism and/or gastrointestinal function of the host in a manner which is beneficial to good health. This less rigid concept of essentiality or indispensability gives due recognition to foods as being much more than vehicles of 'essential' nutrients. It also suggests that optimum health might be difficult to achieve by simply ingesting mixtures of such nutrients obtained from the chemist. Nevertheless, some nutrients, e.g. the well-known hypocholesterolaemic effects of niacin and the more controversial effects of vitamin $\mathrm{E}$ and other antioxidants on chronic disease, may confer specific health benefits when taken in pharmacological or non-physiological doses. Such actions may have little in common with those traditionally associated with an essential nutrient, nor do they lie comfortably within the concept of optimum nutrition.

The brief, however, of the authors of the papers in the present symposium was not to try to define optimum nutrient intakes, but rather to indicate ways of determining optimum nutrient status. There are a number of potential indicators of optimum nutrient status. These indicators include balance studies, measurement of mobilizable body stores, tissue saturation studies and the use of functional indices. Only with the latter can the potential levels of nutrient activity be fully explored.

The increasing use of functional indices to determine optimum nutrient status has been driven by new knowledge of the functions of nutrients at the biochemical, physiological and genetic levels. A functional index of nutrient status can be defined as a biochemical, physiological or genetic factor which is: (1) related to function in target tissue(s); (2) sensitively (and, preferably, specifically) affected by changes in dietary intakes or stores of that nutrient. Optimum nutrient status may be defined as when a functional index reaches a certain quantitative value at which it is no longer affected by intakes or stores of that nutrient. These definitions cover the exploration of nutrient activity in both directions, i.e. from the nutrient status associated with the prevention of overt deficiency disease or 
from the nutrient status associated with toxic symptoms. Functional indices can be classified further into those which are measures of discrete functions of a nutrient and those which are measures of general functions of a nutrient (Turnlund, 1994).

A discrete functional index often relates to the first limiting biochemical system, and should intuitively provide the most valid index of optimum nutrition for a particular nutrient. For many nutrients the first limiting biochemical system is unknown or not readily measured or accessible. Moreover, maximizing (or minimizing) the activity of a biochemical or physiological system may not necessarily be desirable if changes in the system cannot be shown to have an impact on health and well-being.

A general functional index can be more accessible for measurement and more directly related to disease mechanisms or ill health than a discrete functional index. Examples of general functional indices, which could prove useful for indicating optimum nutrient status, are: immune function, antioxidant status, glucose tolerance, haemostasis, bone health, arterial compliance, muscle strength, blood pressure, DNA repair, work capacity, BMR and cognitive performance. Stressors such as exercise, temperature and mental tasks can also be used to aggravate detrimental changes in a functional index at a particular nutrient status (Lukaski \& Penland, 1996). General functional indices, however, by their very nature are usually less specific than discrete functional indices. Studies must be designed so that a cause and effect relationship is established not only between nutrient status and the functional index but also between nutrient status and ill health. Given the daunting problems with nutritional research, design and implementation of such studies will undoubtedly be difficult and timeconsuming, even when candidate functional indices have been identified for particular nutrients.

The papers that follow aim to give the current state of knowledge with respect to the use of functional indices to define optimum nutrient status for the traditional nutrients, dietary fibre and some phytochemicals. Attempts have been made to give some coherence to the various groupings of these nutrients, but obviously the relevant expertise of the invited authors was the final determinant of the scope of each paper.

\section{References}

Combs GF (1996) Should intakes with beneficial actions, often requiring supplementation, be considered for RDAs? Journal of Nutrition 126, 2373S-2376S.

Lukaski HC \& Penland JG (1996) Functional changes appropriate for determining mineral element requirements. Journal of Nutrition 126, 2354S-2364S.

Turnlund JR (1994) Future directions for establishing mineral/trace element requirements. Journal of Nutrition 124, 1765S-1770S. 